\title{
In a Rat Model of Parkinsonism, Lesions of the Subthalamic Nucleus Reverse Increases of Reaction Time but Induce a Dramatic Premature Responding Deficit
}

\author{
Christelle Baunez, André Nieoullon, and Marianne Amalric \\ Laboratoire de Neurobiologie Cellulaire et Fonctionnelle, CNRS, 13402 Marseille Cedex 20, France
}

Lesions of the subthalamic nucleus (STN) have been found to reduce the severe akinetic motor symptom produced in animal models of Parkinson's disease, such as in monkeys treated with 1-methyl-4-phenyl-1,2,3,6-tetrahydropyridine (MPTP) or in monoamine-depleted rats. However, little is known about the effect of STN exclusion on subtle motor deficits induced by moderate dopaminergic lesions in complex motor tasks. The present study was thus performed on rats trained in a reaction time (RT) task known to be extremely sensitive to variations of dopamine transmission in the striatum. Animals were trained to release a lever after the onset of a visual stimulus within a time limit to obtain a food reward. Discrete dopamine depletion produced by infusing the neurotoxin 6-hydroxydopamine (6-OHDA) bilaterally into the dorsal part of the striatum, produced motor initiation deficits which were revealed by an increase in the number of delayed responses (lever release after the time limit) and a lengthening of RTs. In contrast, bilateral excitotoxic lesion of the STN with ibotenic acid induced severe behavioral deficits which were opposite to those produced by the dopaminergic lesion, as shown by an increase in the number of premature responses (lever release before the onset of the visual stimulus) and a decrease of RTs. Surprisingly, the performance of the animals bearing a double lesion (striatal dopaminergic lesion followed $14 \mathrm{~d}$ later by STN ibotenic lesion) was still impaired $40 \mathrm{~d}$ after the ibotenic lesion. As expected, the 6-OHDA-induced motor initiation deficits were reversed by a subsequent STN lesion. However, the dramatic increase of premature responses contributing to major behavioral deficits induced by the STN lesion remained unchanged. Thus, the bilateral lesion of the STN was found to alleviate the motor deficits in this model of parkinsonism, but essentially produced over time, long lasting deficits that might be related to dyskinesia or cognitive impairment. The present results strongly support the recent concept of a

\footnotetext{
Received Mar. 15, 1995; revised June 2, 1995; accepted June 6, 1995

This study was supported by grants from the CNRS, the "Université de la Méditerranée" (Aix-Marseille II) (Ministère de l'Enseignement Supérieur et de la Recherche) and DRET (Convention 91.34.157.00.470.75.01). C.B. was supported by a grant from the French Government (Ministère de l'Enseignement Supérieur et de la Recherche, Contrat 92221). We thank Dr. L. KerkerianLeGoff for scientific discussion and help in performing the immunostaining experiments, D. Terramorsi for taking care of the animals, A. Ivinec for pholographic work, and C. Decavèle for english corrections of the manuscript.

Correspondence should be addressed to Marianne Amalric, Laboratoire de Neurobiologie Cellulaire et Fonctionnelle, CNRS, 31 Chemin J. Aiguier, 13402 Marscille Cedex 20, France.

Copyright (C) 1995 Society for Neuroscience $0270-6474 / 95 / 156531-11 \$ 05.00 / 0$
}

predominant control of the STN on basal ganglia output structures.

[Key words: subthalamic nucleus, dopaminergic lesion, reaction-time task, rat, parkinsonism, basal ganglia disorders]

In Parkinson's disease, a progressive degeneration of the dopaminergic (DA) neurons projecting from the substantia nigra to the striatum results in akinesia (difficulties to initiate movements), muscular rigidity and tremor. The clinical deficits observed in patients suffering from such a disease primarily have been largely considered to result from a decrease in the DA transmission at the striatal level (Bernheimer et al., 1973). Although treatment with dopamine agonists is efficacious (i.e., Levodopa), it is in fact associated over time with various side effects such as dyskinesia, motor fluctuations ("on-off" phenomenon) and even psychosis. Recent advances in the knowledge of basal ganglia anatomo-functional organization (Albin et al., 1989; DeLong, 1990) has given new insights into the search for possible alternative therapies. For example, disruption of DA transmission into the striatum is thought to result in subthalamic nucleus (STN) overactivity. Since the STN sends major excitatory amino acid (FAA) containing projections to the internal globus pallidus (GPi or entopeduncular nucleus (EP) in the rat) and the substantia nigra pars reticulata ( $\mathrm{SNr}$ ) (Smith and Parent, 1988; Brotchie and Crossman, 1989; Robledo and Feger, 1990), such an overactivity of the STN may likely induce hyperactivity in basal ganglia output structures. Consequently, suppression of the nigrostriatal DA system is supposed to indirectly induce an increased inhibition of the thalamo-cortical neurons. Subsequent reduced activation of the cortex would thus presumably be responsible for some of the parkinsonian troubles (see DeLong, 1990).

Confirmation of this hypothesis comes from an increase in the uptake of 2-deoxyglucose as an index of regional neuronal activity in the STN (Mitchell et al., 1989) and from electrophysiological recordings of the neuronal activity in the STN or in its output structures both in MPTP-treated monkeys (Bergman et al., 1990, 1994; Filion and Tremblay, 1991) or in monoaminedepleted rats (Robledo and Feger, 1990). Various experimental procedures, ranging from excitotoxic lesion of the STN (Bergman et al., 1990; Graham et al., 1990; Aziz et al., 1991; Brotchie et al., 1991; Anderson et al., 1992; Benazzouz et al., 1993) to the pharmacological blockade of the excitatory (probably glutamatergic) projection in the output structures of the STN (Klockgether and Turski, 1990; Klockgether et al., 1991; Burns et al., 1993) have been used in order to counteract the hyper- 
activity of the STN observed after DA depletion. The data in general support the hypothesis of a beneficial influence of the STN exclusion in improving the behavior of animals with impaired striatal DA transmission. Finally, the electrical STN stimulation at high frequency, which hypothetically also blocks STN activity, was also shown to improve the behavior in MPTP-treated monkeys (Benazzouz et al., 1993) and to allow a significant reduction of the doses of L-DOPA administered to parkinsonian patients (Benabid et al., 1994). Interestingly, hemorrhage or hematoma at the level of the STN was found to alleviate some of the motor deficits in patients suffering from Parkinson's disease (Sellal et al., 1992; Yamada et al., 1992).

However, in the animal models of parkinsonism, most of the interactions with the STN has been found by studying motor parameters in simple behavioral paradigms, such as rotation in the rat or spontaneous activity in the monkey. In contrast, the involvement of the STN in the control of movement has been the topic of only a few studies in otherwise intact monkeys without previous MPTP treatment by means of cell activity recordings or lesions (Hamada and DcLong, 1992a,b; Dormont et al., 1994; Wichmann et al., 1994a). To further investigate the role of STN in motor control, we thus proposed to test the effects of a bilateral ibotenic lesion of the STN on basal locomotor activity and on behavioral performance of intact rats trained in a reaction time (RT) task. To explore the efficacy of STN lesion in improving motor disability in parkinsonian patients, the effects of the same lesion were then tested in rats with a previous DA denervation of the striatum known to induce only moderate deficits in the RT task (Amalric and Koob, 1987). In this task, the animals were required to depress a lever and wait for a visual trigger stimulus before releasing it as fast as possible. This task was previously found to be very sensitive to a discrete variation in the level of DA activity in the striatum (Amalric and Koob, 1987; Amalric et al., 1995; Baunez et al., 1995).

\section{Material and Method}

\section{Animals}

Male Wistar rats ( $n=49$, Charles River, France), weighing $110-120$ $\mathrm{gm}$ at the beginning of the experiment, were housed three to four per cage and maintained on a $12 \mathrm{hr}$ light/dark cycle (7:00 A.M.-7.00 P.M.). Food supply was restricted to $15-17 \mathrm{gm} / \mathrm{rat}$ per day in order to keep the animals' weight at $80 \%$ of that of free-feeding animals. Water was provided ad libitum, except during experimental sessions.

\section{Behavioral tests}

Reaction time task. The animals were first trained to press a lever down for food reinforcement in standard operant boxes (Campden Ltd., U.K.). The retractable lever required a force of 0.8 newtons for switch closure. A stimulus light ( $2.8 \mathrm{~W}$ hulh) was located above the lever. On the final program, rats were trained to depress the lever and wait for the onset of the light-cue visual conditioning stimulus (CS) presented after four randomly and equiprobably generated intervals $(500,750,1000$, and $1250 \mathrm{msec}$ ). To be rewarded by a food pellet (Bioserv Inc., NJ) the rats had to release the lever as fast as possible within an arbitrary time limit set at 600 insec by the experimenter. The reaction time (RT) was taken to be the time elapsing from the CS onset to the lever release. One daily session ended after 100 trials. Performance was evaluated by recording the number of correct responses and incorrect (nonrewarded) responses which were divided into two categories: "premature response," when the lever was released before the onset of the CS, or "delayed response," when the lever was released after the time limit of 600 msec. For each correct and delayed response, RT was recorded and led to subsequent distribution analysis.

Locomotor activity. The locomotor activity was measured in eight wire and Plexiglas cages $40 \times 25 \times 23 \mathrm{~cm}$, with two horizontal IR photocell beams located across the long axis of the cage (Imctronic, France). Beam interruptions were accumulated over $1 \mathrm{~min}$ intervals and recorded for each minute bin by means of a computer (Tandon PCA 12SL).

\section{Experimental procedure}

After stabilization of the behavioral performance, the preoperative baseline values were recorded at five consecutive sessions. The rats were arbitrarily divided into two groups with an equal proportion of rats with moderate to good performance $(50-80 \%)$, and subjected to a bilateral striatal vehicle (sham group $n=22$ ) or 6-OHDA injection (lesion group $n-27$ ). After a recovery period of 1 week, all the animals were tested again for seven consecutive sessions. A second surgery was then carried out. Within each group, half of the animals were subjected to a bilateral subthalamic vehicle injection ("double sham animals," $n=9$ and " 6 OHDA lesioned and sham STN animals," $n=12$ ). The other half were subjected to a bilateral injection of ibotenic acid into the STN " 6 OHDA sham and STN lesioned animals," $n=13$ and "double lesioned rats," $n=15$ ). All the animals were tested again for 27 sessions over 6 weeks post STN-lesion ( $8-40 \mathrm{~d}$ postlesion).

On the 5th and 17th days after the STN lesion, a small number of animals belonging either to the "double sham group" $(n=4)$ or the "STN lesion group" $(n=4)$ was tested in locomotor activity cages for $2 \mathrm{hr}$ before being tested on the RT task. Additional nonquantitative behavioral observations of the animals in their home-cages were performed during the first week following lesion.

\section{Lesion surgery}

6-OHDA lesion. The animals were anaesthetized intramuscularly with a solution of xylazine $(15 \mathrm{mg} / \mathrm{kg}) / \mathrm{ketamine}(100 \mathrm{mg} / \mathrm{kg})$ and secured in a Kopf stercotaxic instrument with the tooth bar set $+5.0 \mathrm{~mm}$ above the interaural line. Lesions were made by a bilateral injection of 6-hydroxydopamine $(4 \mu \mathrm{g} / \mu \mathrm{l}, 2 \mu \mathrm{l} / \mathrm{side})$ into the striatum at the following coordinates from the atlas of Pellegrino et al. (1979): AP $+1.8 \mathrm{~mm}, \mathrm{~L}$ $\pm 3.5 \mathrm{~mm}, \mathrm{DV}-5.0 \mathrm{~mm}$. The sham group received the solvant of the 6-OHDA solution (i.e., ascorbate $0.1 \mathrm{mg} / \mathrm{ml}$ in $0.9 \%$ saline) at the same coordinates.

Ibotenic acid lesion. Two weeks after the first surgery, each group was subjected to the same surgical procedure carried out with the tooth bar set $-2.7 \mathrm{~mm}$ above the interaural line. The animals received a bilateral injection of $0.5 \mu \mathrm{l}$ of ibotenic acid $(9.4 \mu \mathrm{g} / \mu \mathrm{l}$, i.e., $53 \mathrm{~mm})$ into the subthalamic nucleus at the following coordinates taken from the atlas of Paxinos and Watson (1986): $\mathrm{AP}-3.8 \mathrm{~mm}, \mathrm{~L} \pm 2.4 \mathrm{~mm}$, DV $-8.35 \mathrm{~mm}$ from the skull. Sham groups received the injection of phosphate buffer $(0.2 \mathrm{M})$ at the same coordinates.

\section{Histology}

After completion of the behavioral test, all the animals were sacrificed and a tyrosine hydroxylase immunostaining was performed in order to estimate the extent and location of the DA depletion in the striatum and the SN. The animals were anaesthetized with chloral hydrate $(400 \mathrm{mg} /$ $\mathrm{kg}, \mathrm{i} . \mathrm{p}$ ) and perfused with $500 \mathrm{ml}$ of $4 \%$ paraformaldehyde in $0.12 \mathrm{M}$ phosphate buffer (pII 7.4) through ascending aorta. The brains were removed and postfixed overnight at $4^{\circ} \mathrm{C}$ in the same fixative. Then, they were sectioned using a vibratome (LANCER). Frontal 30-40 $\mu \mathrm{m}$ thick sections of the striatum were collected in a $0.5 \mathrm{M}$ Tris-saline buffer, $\mathrm{pH}$ 7.6. All sera were prepared in the Tris-saline buffer and all steps of the immunohistochemical procedure, previously described (Kerkerian et al., 1988), were carried out at room temperature, except for the incubation of the primary antiserum performed at $4^{\circ} \mathrm{C}$. After reaction with $3,3^{\prime}$ diaminobenzidine $(0.05 \%$, tetrahydrochloride, Sigma) and hydrogen peroxide $(0.012 \%)$, the slices were mounted on gelatine-coated slides and observed with a light-microscope in order to assess the extent of the lesion. Sections collected at the level of the substantia nigra were kept in order to examine whether the dopaminergic soma were degenerated after the 6-OHDA injection into the striatum.

A Nissl detection of gliosis reaction was performed in the STN lesioned animals to further detect the extent and location of the ibotenic lesion. Frontal $50 \mu \mathrm{m}$ thick brain sections were collected at the level of the subthalamic nucleus. They were then stained with cresyl violet. Only rats showing a bilateral lesion within the STN area were included in the data analysis.

\section{Data and statistical analysis}

The data were analyzed using the Clear Lake Research analysis of variance (CLR ANOVA) program. The results are expressed as means for each 
of the motor parameters (i.e., correct, premature, delayed responses per session and RTs for correct trials) in the four various groups of animals. Means were obtained at every pre- and postlesion session.

During a representative session of the pre- (day 5 before lesion) and postsurgical days (day 12 after 6-OHDA or STN lesion), RTs (for correct and delayed responses) longer than $50 \mathrm{msec}$ and shorter than 800 msec fitting a normal distribution were analyzed. The general distribution of RTs was analyzed by summing up the number of trials with RT at every interval chosen (i.e., $50 \mathrm{msec}$ ).

The performance in the first two sessions following the dopaminergic lesion was not statistically analyzed (and not presented in the results) because some of the animals $(n=5)$ were still impaired and did not complete the 100-trial session. The total of the 35 sessions was thus divided into 7 periods (i.e., weeks) consisting of 5 consecutive sessions. The first period was taken to be the five sessions preceding the first surgery (i.e. baseline period). The second period comprised the five sessions recorded after the 6-OHDA surgery (10th to 14th day). The third, fourth, fifth, sixth, and seventh periods coincided respectively with the second, third, fourth, fifth, and sixth week following the STN surgery. All these data were subjected to a three-way ANOVA with the different groups (the four various experimental groups) forming the between-group factor, the seven periods (weeks) of five consecutive sessions and the five consecutive sessions by period forming the two within factors. After a significant global ANOVA, subsequent ANOVAs were performed for each group separately with two within-factors (seven levels for the seven periods and five levels for the five consecutive sessions), followed by post-hoc comparisons using paired $t$ test after significant ANOVA. For each period of five consecutive sessions, another ANOVA was carried out with the four groups as the between-factor and the five sessions as the within-factor, followed by post-hoc comparisons using the Newman-Keuls test, if appropriate. The criterion of significance was taken to be $95 \%$ or more.

\section{Results}

\section{Histological examination}

Extension of the bilateral ibotenic lesions of the STN was determined after examination of the brain slices at the level of the STN in all animals. As compared to sham-operated animals (Fig. $1 A$ ), the lesioned animals showed marked gliosis and a loss of neurons within the STN (Fig. 1B). The STN lesion was shown to be restricted to the medial part of the nucleus in most of the cases, without affecting the nearby structures. In some subjects, however, the lesion appeared to be unilateral only or bilaterally located above the STN $[n=3$ of the 6-OHDA-sham group ("STN lesion" group), $n=7$ of the 6-OHDA-lesion ("double lesion" group)]. These lesions did not either modify the performance of the "STN lesion" animals or maintained the 6-OHDA-induced deficits of the "double lesion" group (data not illustrated). The performance of these animals were discarded from statistical analysis.

The brain slices of the 6-OHDA lesion group were examined and compared to those of the control animals. The lesion extended from the rostral to the caudal part of the striatum, with a variable extension to the extreme rostral part and the largest extension in the caudal part. Nucleus accumbens and olfactory tubercle DA innervation remained intact. Furthermore, a degeneration of the dopaminergic soma was observed at the level of the medial region of the substantia nigra sparing the pars lateralis where some TH-immunoreactive neurons could be found (not illustrated). The striatal dopaminergic lesion extended mostly to the lateral part of the striatum as shown by the loss of TH immunoreactivity in a representative animal of the lesion group (Fig. $2 B$ ) as compared to a sham-operated animal, showing a dense TH immunostaining throughout the striatum (Fig. 2A).

\section{Behavioral results}

The preoperative baseline performance of the various groups measured during five consecutive daily sessions amounted up to
$60 \%$ correct responses on average per session (Fig. 3). This level obviously depended on the number of premature and delayed responses, averaging approximately $30 \%$ and $10 \%$, respectively, per session. No significant difference between groups was revealed by statistical analysis performed on the percentage of correct responses before surgery.

\section{Control group}

Performance of the "double-sham lesioned animals" was stable throughout the whole experiment as illustrated in Figure 3. The proportion of correct and incorrect responses did not change significantly over time during the total of 35 sessions tested ( $p$ $>0.05, \mathrm{NS}$ ).

\section{Effects of the STN ibotenic lesion on spontaneous and conditioned behavior}

Locomotor activity. No obvious motor deficit (i.e., that could be related to the ballism symptom observed in human and nonhuman primates) could be observed in the animals bearing STN ibotenic lesion. The animals behaved socially in their home cages and could eat and drink on their own. For some of these animals $(n=8)$, the locomotor response was measured in photocell activity cages on day 5 and 17 after the STN lesion. Five days after the STN lesion, the level of spontaneous locomotor activity of the lesioned animals showed a nonsignificant tendency to increase during the first 30 min of testing, when compared to the sham-operated animals. This tendency disappeared, however, when the animals were tested again $17 \mathrm{~d}$ after the lesion (data not illustrated).

Reaction time performance. Excitotoxic lesion of the STN was shown to induce a severe deficit in the RT task. As illustrated in Figure 3, the number of correct responses of the lesioned animals was dramatically decreased over the 5 weeks tested after the lesion as compared to their preoperative values $\left(p<0.01\right.$, paired $t$ test, after significant ANOVA, $F_{(6,54)}=23.6$ ) and to the performance of the sham-operated animals $(p<0.01$, Newman-Keuls after significant ANOVA, at each of the 5 weeks of testing following the STN lesion, $F_{(3.35)}=19.5,12.4,8.7,9.5$, and 7.6 , respectively). The impairment in the number of correct responses resulted from a dramatic increase in the number of premature responses, as compared to the baseline of the same animals $\left(p<0.01\right.$, paired $t$ test after significant ANOVA, $F_{(6,54)}$ $=17.1)$ and of the sham-operated animals (control group) $(p<$ 0.01 , Newman-Keuls after significant ANOVA for each of the 5 test weeks $F_{(3,35)}=15.5,11,8.5,10.6$, and 7.9 , respectively). The number of delayed responses was slightly increased after the lesion for the second to the fourth week postlesion as compared to preoperative values (Fig. 3). However, a similar effect was also observed in the control group. Therefore, no significant difference between performance in the lesioned and sham-operated animals was found (ANOVA, $p>0.05$, NS for each of the 5 weeks tested after the lesion).

The mean RTs in the lesioned group averaged $310 \mathrm{msec}$ during baseline performance and was not different from RTs measured in control animals (mean RTs $=330 \mathrm{msec}$ ) (Fig. 4A). After the STN lesion, mean RTs were significantly decreased as compared to pre-operative values $(p<0.01$ and $p<0.05$ at the fourth and fifth weeks postlesion, paired $t$ test after significant ANOVA, $F_{(6,54)}=6.13$ ). RTs were also significantly shortened as compared to those of control animals during the first week postlesion only $(p<0.05$, Newman-Keuls after significant ANOVA, $F_{(3,35)}=4.43$ ) (Fig. $4 A$ ). The RT distribution curve, 
Figure 1. Structural damage induced by the STN ibotenic lesion. Cresyl violet stain of a frontal section at the STN level of a sham $(A)$ and a lesioned animal $(B)$. Inset, Schematic frontal section at the STN level (hatched area) $(-3.8 \mathrm{~mm}$ from bregma from the atlas of Paxinos and Watson). Stn, Subthalamic nucleus; $C p$, cerebral peduncle.

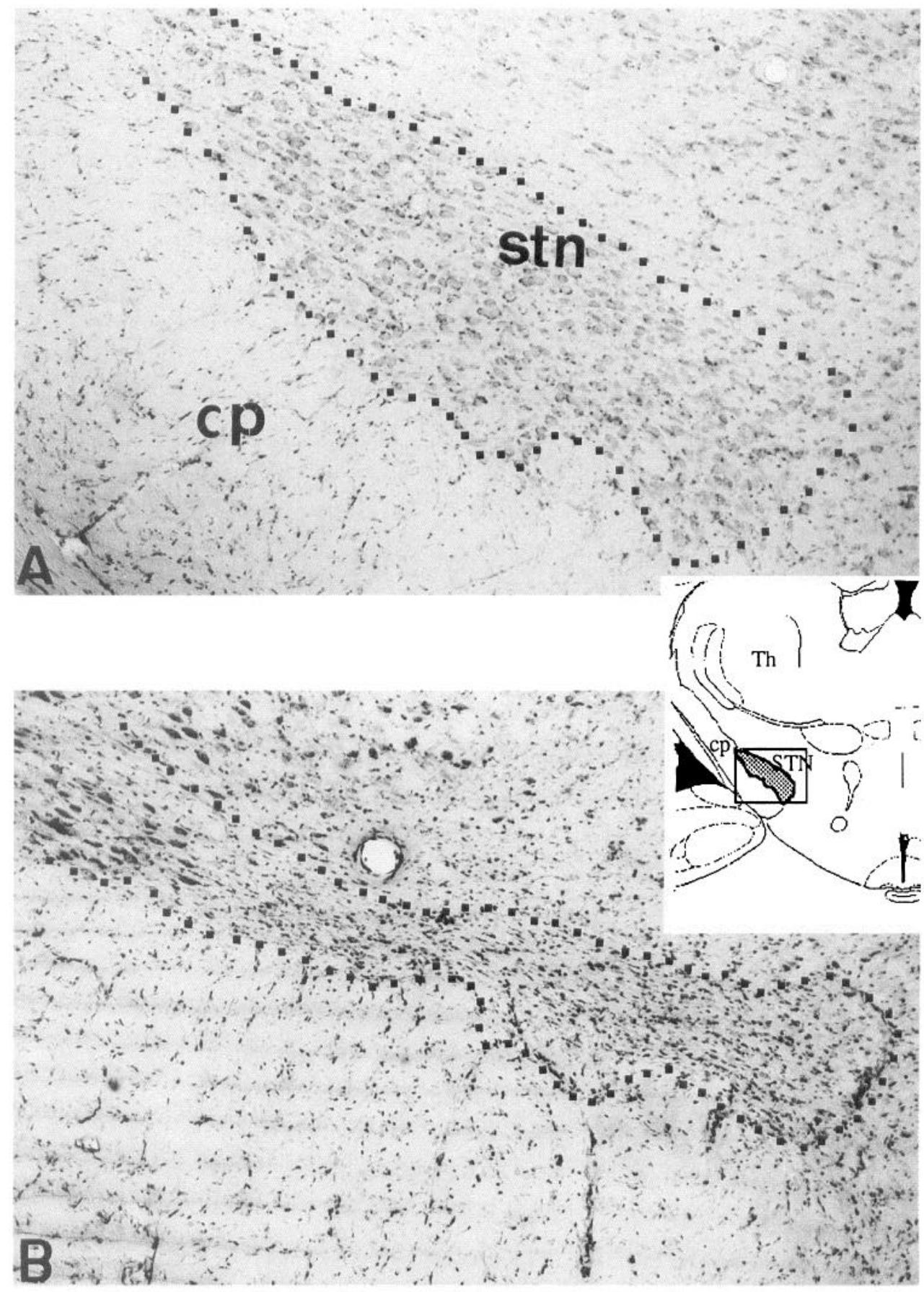

illustrated for four representative animals belonging to the STN lesion group, revealed that the RTs were not shifted to the left to shorter values as compared to the control session, but that they were scattered between lower $(50 \mathrm{msec})$ and higher $(800$ msec) values (Fig. 4B).

\section{Effects of the striatal 6-OHDA lesion on RT performance}

The effects induced by the 6-OHDA infusion into the dorsal part of the striatum were analyzed on postoperative days 10-14 in the "double-lesion" group, prior to the STN lesion. As previously shown (Amalric et al., 1995), they were expressed by a decrease in the number of correct responses (Fig. 5), as compared to their baseline performance ( $p<0.01$, paired $t$ test after significant ANOVA, $\left.F_{(6,42)}=14.3\right)$ and to that of sham-operated animals $(p<0.05$, Newman-Keuls after significant ANOVA for the postoperative period from days $\left.10-14, F_{(3,35)}=5.65\right)$. The dopaminergic lesion did not affect the number of premature responses as compared to the baseline ( $p>0.05$, NS paired $t$ test after significant ANOVA within the same group) or to the control group performance $\left(F_{(3,35)}=1.1, p>0.05\right)$. In contrast, the major deficit observed was a significant increase in the number of delayed responses, as compared to baseline $(p<0.01$, paired $t$ test after significant ANOVA, $F_{(6,42)}=3.8$ ) or to control group performance $(p<0.01$, Newman-Keuls after significant ANOVA, $F_{(3,35)}=7.4$ ). Within the same group (Fig. $6 A$ ), the mean RTs averaging $310 \mathrm{msec}$ before surgery was significantly increased up to $360 \mathrm{msec}$ after the lesion $(p<0.01$, paired $t$ test after significant ANOVA, $\left.F_{(6,42)}=4.34\right)$ and appeared signifi- 

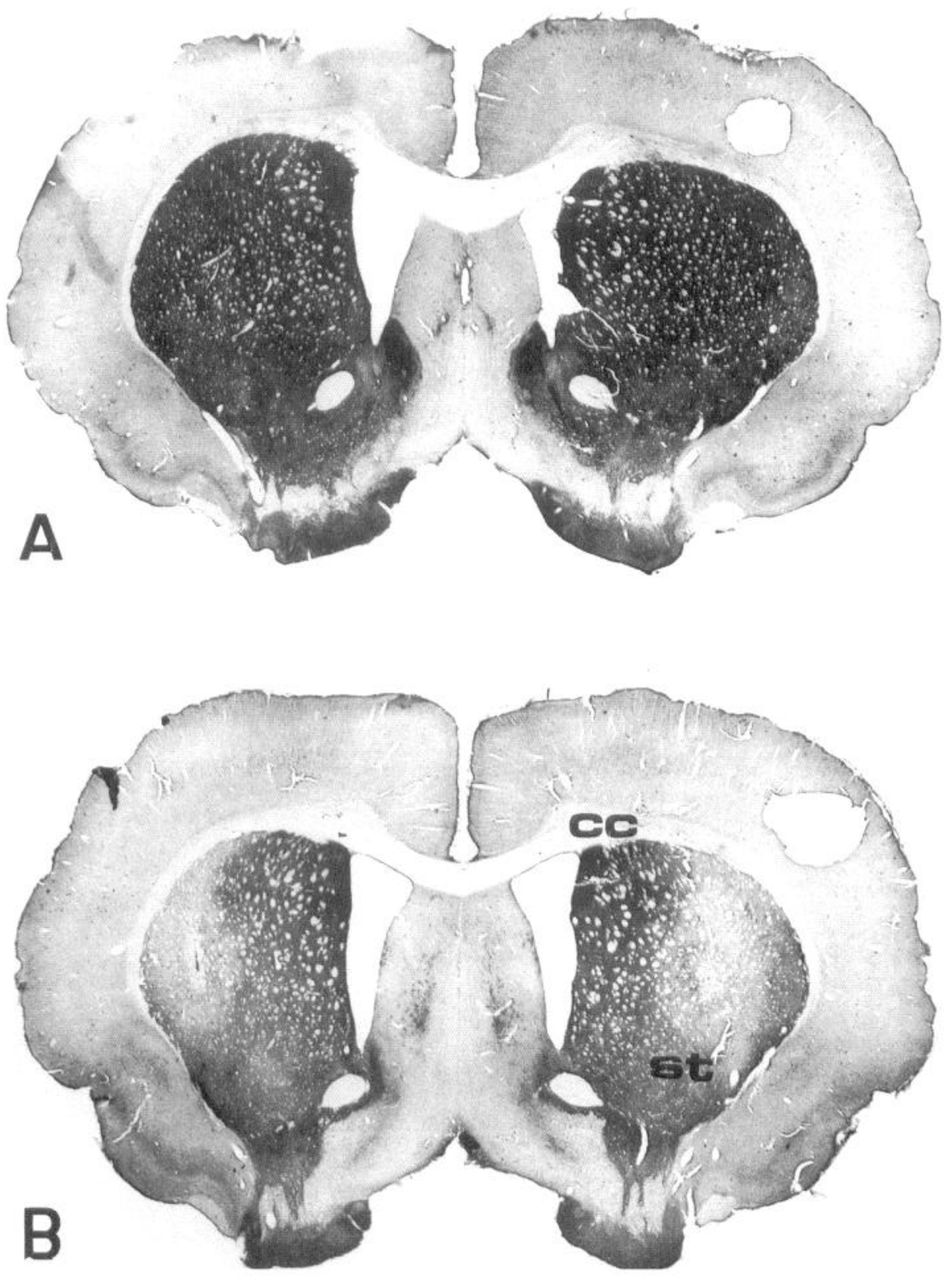

Figure 2. Frontal sections showing exemples of striatal dopaminergic innervation detected with TH-immunostaining in sham $(A)$ and 6-OHDA lesioned representative animals $(B)$. The intrastriatal injection of 6-OHDA induced a DA denervation which was in general restricted to the dorsolateral striatum and spared the nucleus accumbens and olfactory tubercule. $c c$, Corpus callosum; st, striatum. cantly different from RTs of control animals ( $p<0.05$, Newman-Keuls after significant ANOVA, $F_{(3,35)}=3.03$ ). This effect is illustrated for two representative subjects in Figure $6 B$ (left) by a shift to the right of RT distribution curve.

The long-term effect of the dopaminergic lesion tested in a separate group of animals receiving only a 6-OHDA injection, revealed that the animals remained impaired on RT performance throughout the experiment (i.e., 54th day post-striatal lesion). Recovery of preoperative level in the number of delayed responses was, however, observed at week 6th (after the shamSTN lesion, i.e., 50th to 54th day post-striatal lesion) for some of these animals (data not illustrated).

\section{Effects of combined striatal 6-OHDA and STN ibotenic lesions} on RT performance

As illustrated in Figure 5, from the 2nd to the 6th week after the STN lesion, the performance of the animals with a double lesion was severely impaired. The number of correct responses remained lower as compared to baseline scores $(p<0.01$, paired $t$ test after significant ANOVA, $\left.F_{(6,42)}=14.29\right)$ and to scores of the control group (i.e., "double-sham operated animals") $(p<$ 0.01 , Newman-Keuls after significant ANOVA, $\left.F_{(3,35)}=19.48\right)$. The severe deficit in the performance appeared to result from a dramatic increase in the number of premature responses, as compared to baseline ( $p<0.01$, paired $t$ test after significant ANOVA, $\left.F_{(6,42)}=9.97\right)$, and to the control group performance $(p<$ 0.01 , Newman-Keuls after ANOVA for each of the 5 weeks tested following the STN lesion, $F_{(3,35)}=15.48,10.97,8.54$, 10.61 , and 7.86, respectively).

Interestingly, the severe deficit observed in the number of delayed responses after the dopaminergic lesion was completely recovered after the subsequent STN lesion. Although the number of delayed responses remained higher than baseline values during the second week following the lesion $(p<0.05$, paired $t$ test after significant ANOVA, $\left.F_{(6,42)}=14.29\right)$, this effect disappeared after the 12th day postlesion. No significant difference 


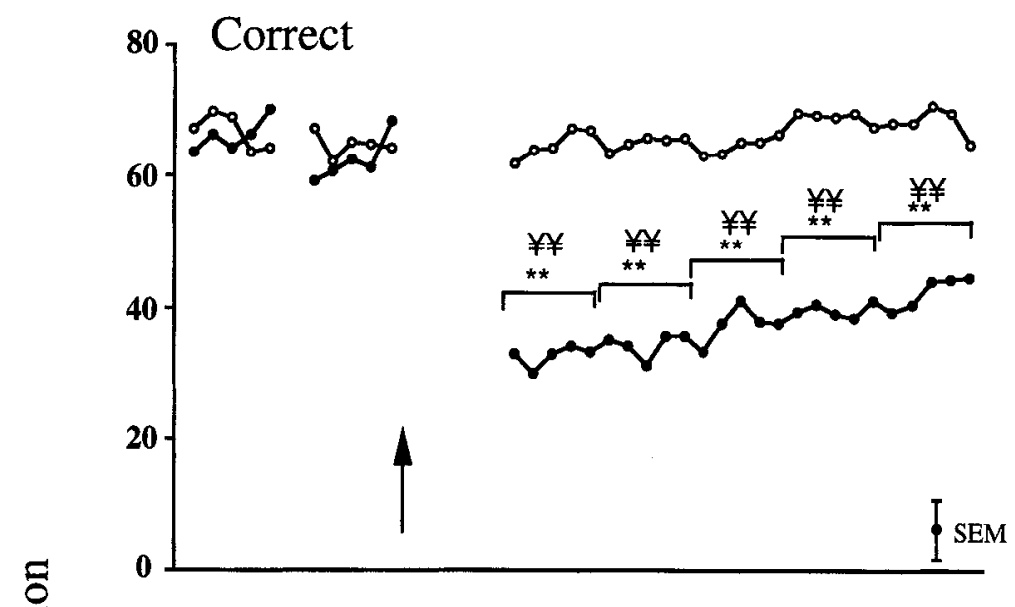

Figure 3. Pre- and postoperative performance of sham-operated (open circles, $n=9$ ) and STN lesioned animals (solid circles, $n=10$ ) in the RT task. The animals were tested $5 \mathrm{~d}$ a week in 100 trial-sessions. Results are illustrated for the 10 preoperative and 25 postoperative sessions (days 8-40) corresponding to weeks $2-6$ after the bilateral ibotenic acid lesion of the STN. Each circle shows the mean number of correct, premature (lever release before the visual stimulus onset), and delayed (lever release after the $600 \mathrm{msec}$ RT limit restriction) responses per session. SEM is calculated at each session and illustrated as a mean of postlesion sessions for clarity purpose. * or **, Significantly different from baseline performance, $p<0.05$ or 0.01 , respectively, paired $t$ test. $¥ ¥$, Significantly different from sham animals, $p<0.01$ Newman-Keuls test.
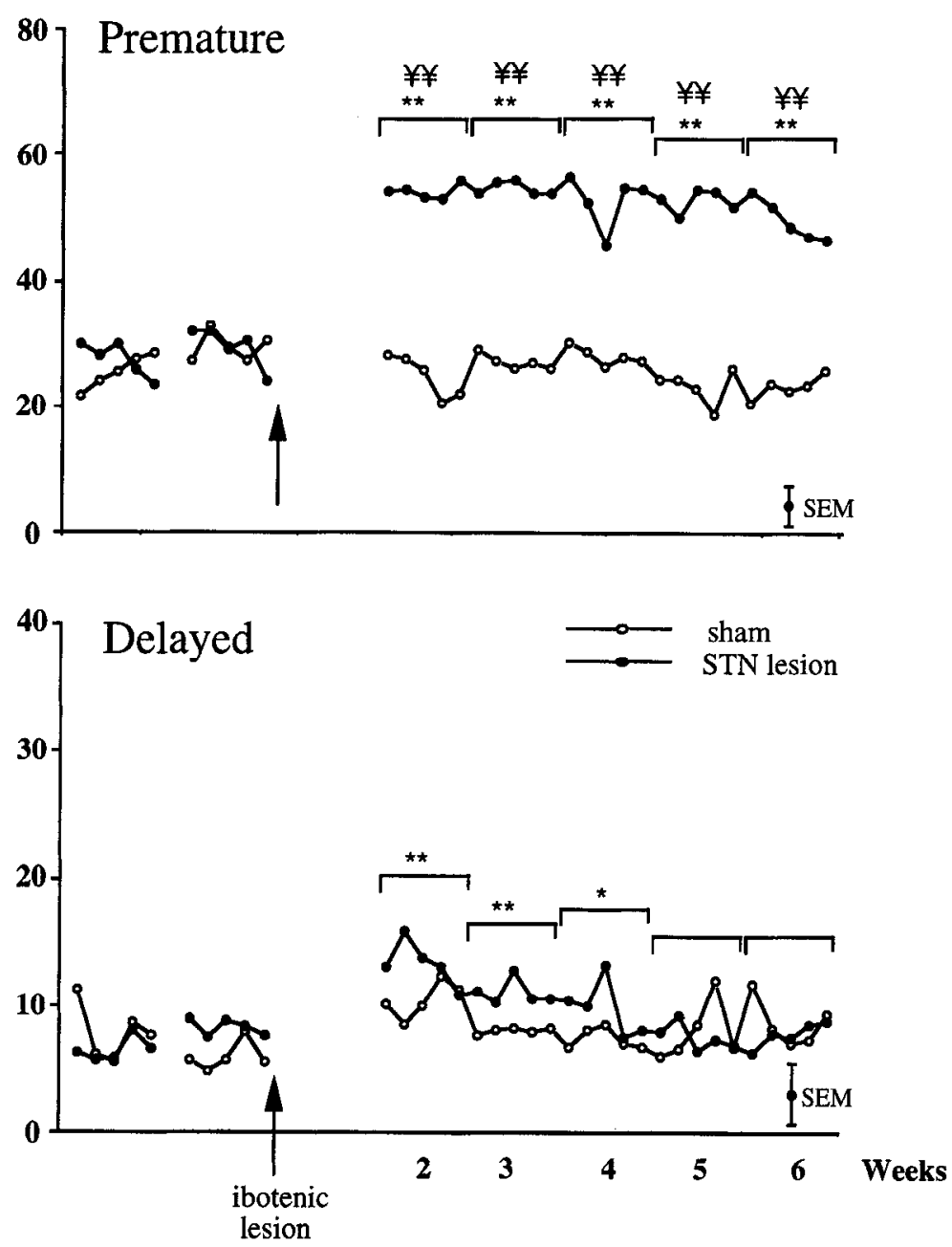

between lesioned and sham-operated animals was found at any time postlesion ( $p>0.05$, NS ANOVA). Furthermore, the lengthening in RTs observed after the 6-OHDA lesion was lowered down to baseline values after the STN lesion, as seen in Figure $6 A(p>0.05$, NS paired $t$ test after significant ANOVA, $F_{(6,42)}=4.34$ and $p>0.05$, NS Newman-Keuls after significant ANOVA, $F_{(3.35)}=4.43$ ). Interestingly, the RTs distribution curve did not shift back towards control values, but the RTs in contrast were found to be scattered between lower and higher values after the subsequent STN lesion (Fig. 6B, right).

\section{Discussion}

The present results show that the bilateral lesion of the STN in rats trained in a reaction-time task severely impaired the behavioral performance. This effect was shown to primarily result from an increased number of premature responses and shortened RTs. In contrast, the bilateral 6-OHDA lesion of the DA terminals in the dorsal striatum area, impaired the performance by increasing the number of delayed responses and lengthening the RTs. Therefore, the STN lesion subsequent to 6-OHDA intrastriatal administration was predicted to reverse the behavioral def- 
A

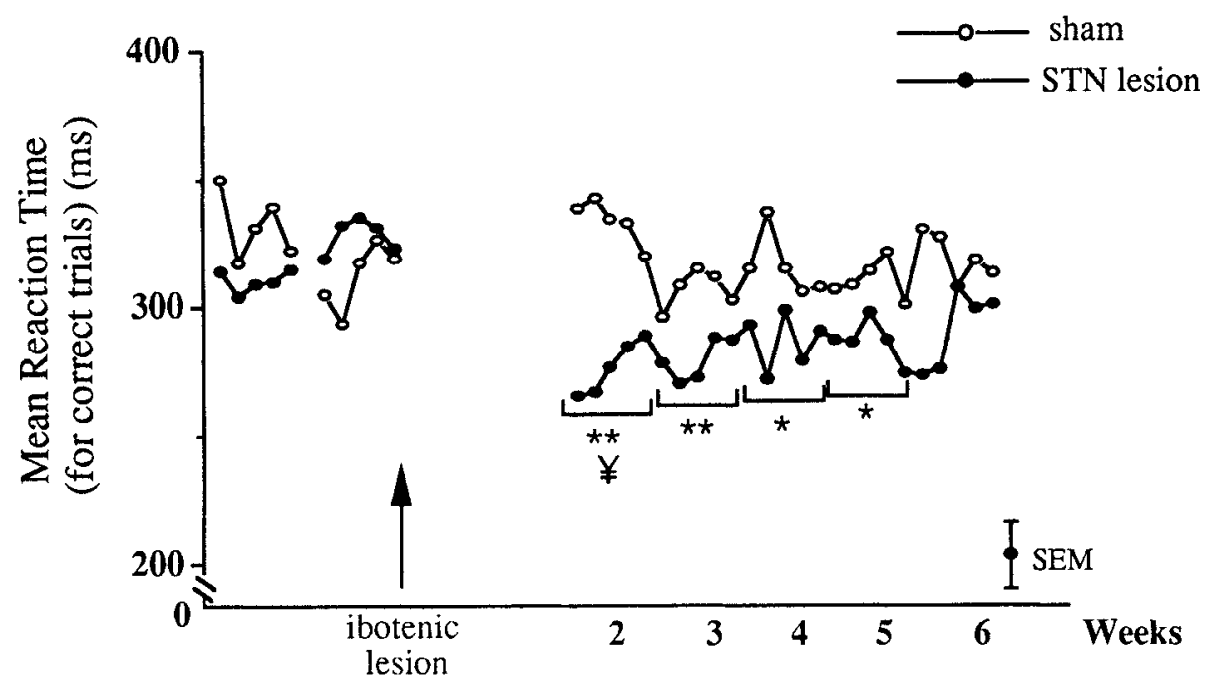

B
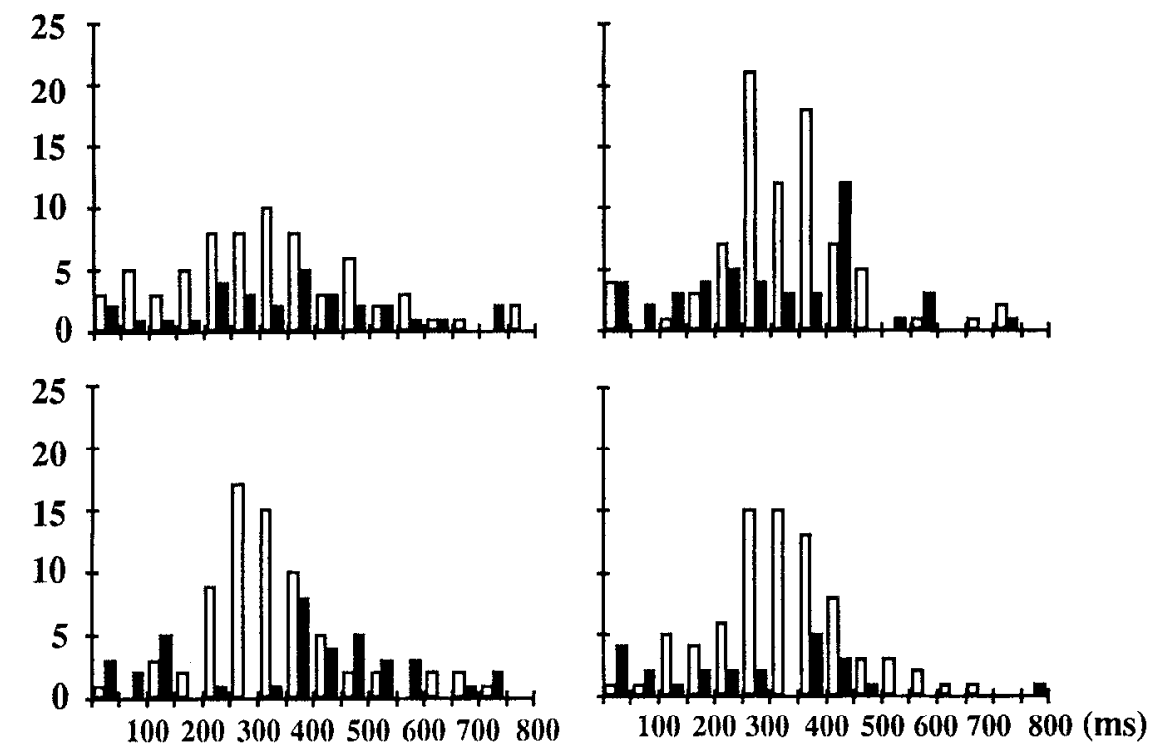

Reaction time distribution
Figure 4. Effects of the STN ibotenic lesion on reaction times. $A$, Mean correct RTs (below $600 \mathrm{msec}$ ) and SEM are illustrated for sham (open circles, $n$ $=9$ ) and lesioned animals (solid circles, $n=10$ ), before (for 10 sessions) and after the STN lesion (for 25 sessions corresponding to weeks $2-6$ postlesion). $B$, Distribution of RTs (longer than $50 \mathrm{msec}$ and shorter than 800 msec) $5 \mathrm{~d}$ before and on day 12 after the lesion, in four representative animals. Each bar represents the number of RTs recorded in $50 \mathrm{msec}$ intervals, during the pre- (open bars) and postlesion session (solid bars). * or **, Significantly different from baseline performance, $p<0.05$ or 0.01 , respectively, paired $t$ test. $¥$, Significantly different from sham animals, $p<0.05$ Newman-Keuls test. icits induced by the dopaminergic striatal lesion. Accordingly, the STN lesion performed 2 weeks after the 6-OHDA striatal infusion was found to reverse the increased number of delayed responses and lengthened RTs. However, the severe deficits induced by the STN lesion (i.e., the increase in the number of premature responses) were maintained, thus suggesting that additive STN lesion to striatal DA depletion did not simply allcviate the behavioral deficit induced by the impairment of striatal DA transmission.

\section{Effects of the STN lesion}

The dramatic increase in the number of premature responses, observed after the STN ibotenic lesion, raised the question as to whether this effect reflects a specific task-related deficit or a spontaneous nonspecific motor hyperactivity which prevents the animals from waiting for the occurrence of the visual stimulus. The slight increase in the spontaneous locomotor activity that was measured in a small number of lesioned animals $5 \mathrm{~d}$ after the lesion was not any longer observed $17 \mathrm{~d}$ after the lesion. Furthermore, no obvious disturbance of spontaneous behavior was observed before the test or when the animals were placed in their home-cages, thus arguing against a general nonspecific hyperactive state. In contrast, the deficits on RT performance remained dramatic throughout the experiment (up to day 40 postlesion). These long-lasting behavioral deficits appeared thus to be specific to the RT task and occurred during the interval preceding the stimulus-light onset, reflecting either a motor activation (i.e., faster processes involved in the initiation of movement) and/or a disruption of more complex processes such as the time estimation or attention.

In view of the functional organization of the basal ganglia proposed by Albin et al. (1989) and DeLong (1990), the lesion of the STN, by reducing the excitatory influence to the striatal output nuclei (GPi and $\mathrm{SNr}$ ), was supposed to increase the tha- 
Figure 5. Pre- and postlesion performance of the animals belonging to the control (open circles, $n=9$ ) or "double lesion" group (solid triangles, $n=$ 8 ) in the RT task. The "double lesion" group was subjected first to a bilateral striatal 6-OHDA lesion followed, $14 \mathrm{~d}$ later, by a bilateral ibotenic acid injection into the STN. Results are illustrated for the 5 preoperative, 5 post- 6 OHDA lesion (corresponding to days $8-14$ postlesion), and 25 post-STN lesion sessions (corresponding to weeks $2-6$, that is, days $8-40$ postlesion). Each sign shows the mean number of correct, premature, and delayed responses per session. SEM was calculated at each session and illustrated as a mean of postlesion sessions for clarity purpose. $*$ or $* *$, Significantly different from baseline performance, $p<$ 0.05 or 0.01 , respectively, paired $t$ test. $¥ ¥$, Significantly different from sham animals, $p<0.01$ Newman-Keuls test.
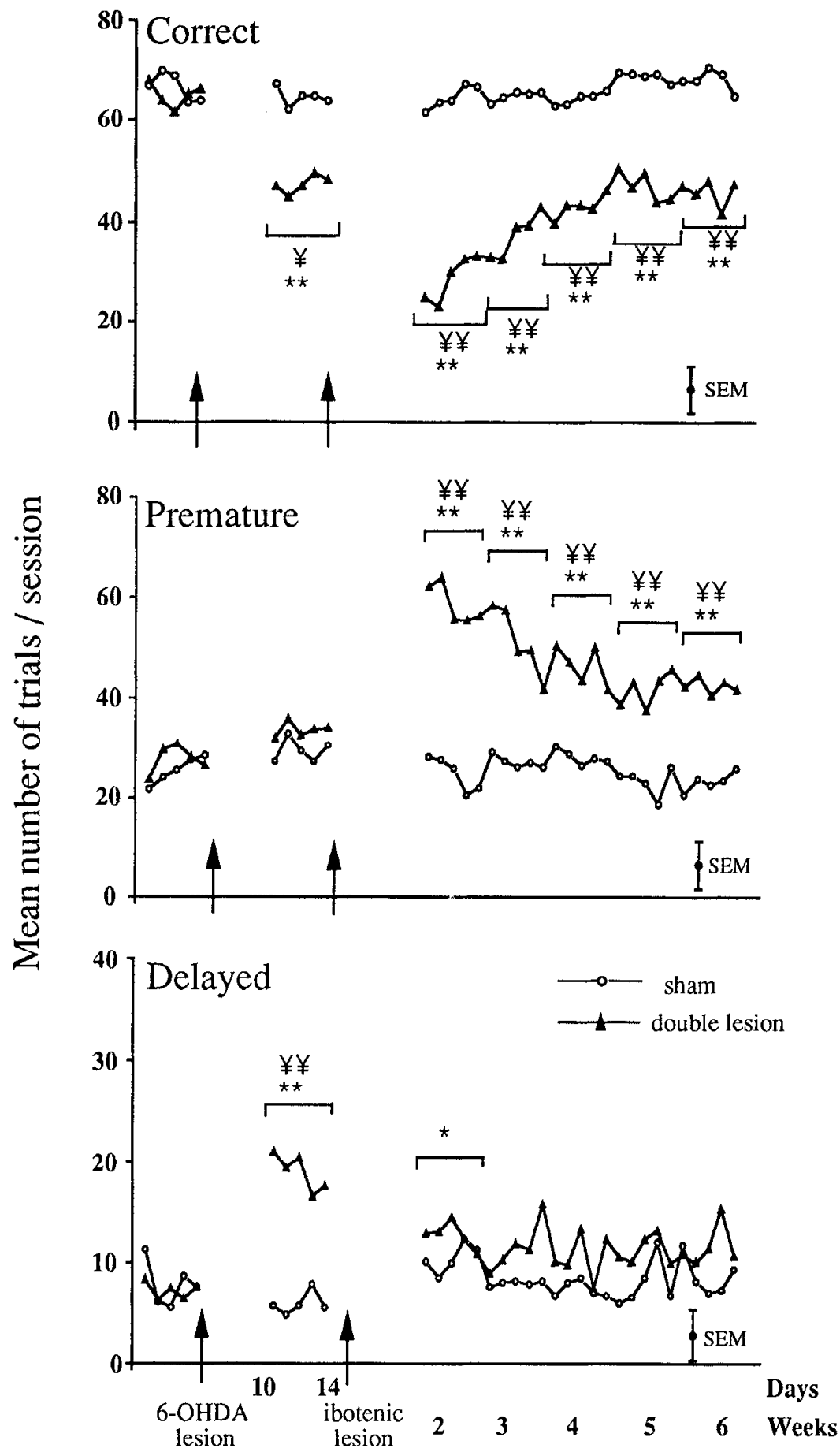

lamic excitatory input to motor cortical regions via a disinhibition process, and hence facilitate the initiation and/or execution of the movement. This hypothesis was supported by electrophysiological studies showing that excitotoxic lesion of the STN in monkeys actually decreases the neuronal activity in the internal segment of the pallidum (Hamada and DeLong, 1992b). Furthermore, the reduction of STN neuronal activity produced by local injection of the GABA agonist muscimol in the STN area, induced a decrease in the neuronal firing at EP level and a decrease in the local cerebral glucose utilization in the same brain area in the rat (Féger and Robledo, 1991; Féger et al., 1991). A reduction of the pallidal GABAergic inhibitory efferent projec- tion could therefore "disinhibit" the thalamo-cortical system, thereby facilitating cortically initiated movements (Albin et al,, 1989; DeLong, 1990). Indeed, behavioral studies have demonstrated that the lesion of the STN or the blockade of its excitatory action on pallidal neuronal activity, using an injection of kynurenic acid (a glutamate receptor antagonist) into the GPi, produces dyskinesia (Robertson et al., 1989) and involuntary movements of the limbs related to ballism in human and nonhuman primate (Crossman, 1987). The incrcased number of premature responses observed in the present reaction-time task after the bilateral lesion of the STN might thus reflect such motor disturbances. This hypothesis appears unlikely, however, be- 
A

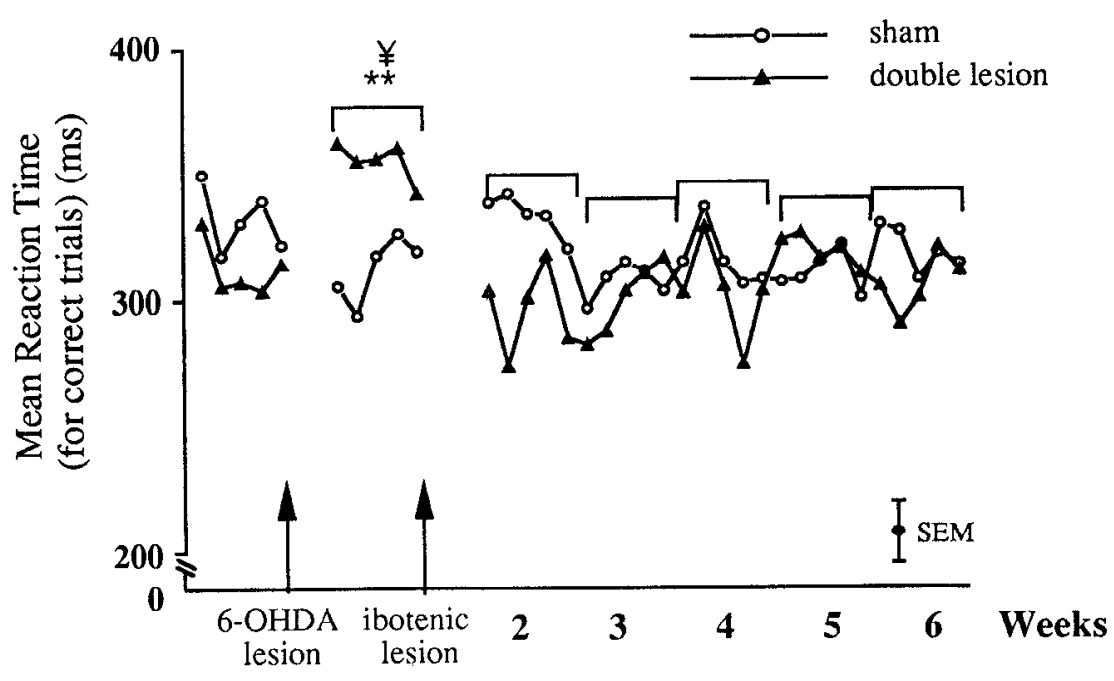

B

Striatal 6-OHDA lesion
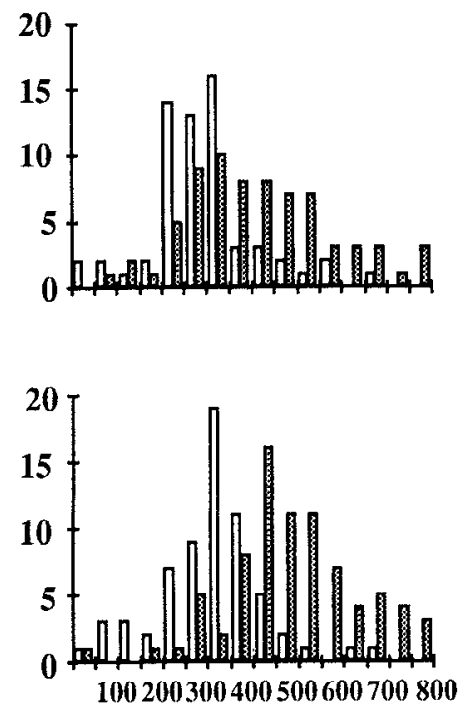

STN lesion
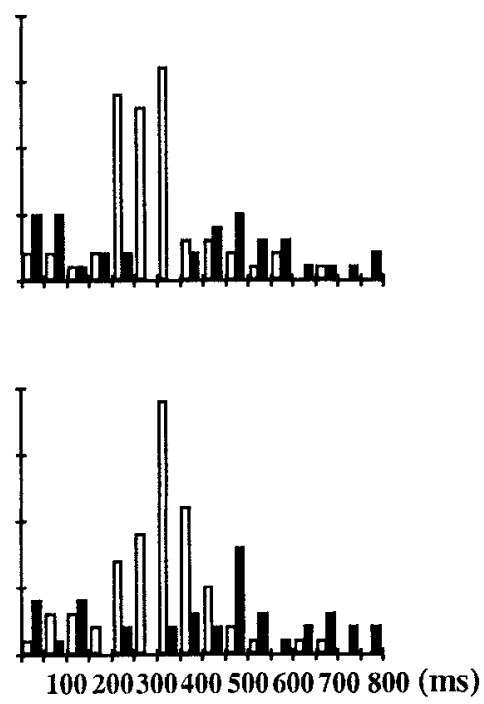

Figure 6. Effects of the STN ibotenic lesion after a striatal 6-OHDA lesion on reaction times. $A$, Mean correct RTs (below $600 \mathrm{msec}$ ) and SEM are illustrated for shanı (open circles, $n-9$ ) and "double lesioned" animals (solid triangles, $n=8$ ), on preoperative and postoperative sessions after 6-OHDA and STN ibotenic lesions (same as Fig. 5). $B$, Distribution of RTs (longer than $50 \mathrm{msec}$ and shorter than $800 \mathrm{msec}$ ) on day 5 before, day 12 after striatal 6-OHDA lesion, and day 12 after STN ibotenic lesions, in two representative animals. Bars represent the number of RTs recorded in each $50 \mathrm{msec}$ interval, during pre- (open bars) and postlesion sessions (dopaminergic lesion, hatched bars, ibotenic lesiun, solid bars).**, Significantly different from baseline performance, $p<0.01$, respectively, paired $t$ test. $¥$, Significantly different from sham animals, $p<0.05$ NewmanKeuls test.

cause the bilateral lesions of the STN in the present study do not impair the execution of the motor task, as the subjects are still able to hold down and release the lever for the total duration of the sessions, excluding any serious motor disturbances. The effect of STN lesion on premature responses and on R'I's is thus rather suggestive of a more complex task-related deficit than an expression of dyskinesia.

A similar effect, expressed by an increase of premature responses and shortened RTs, has recently been found in the same RT task, after pharmacological stimulation of the DA system with dopamine agonists. This effect was attributed to a higher level of motor preparation or "motor readiness," without altering attentional processes (Baunez et al., 1995). Moreover, DA activation was found to induce a shift to the left of the RT distribution curve, suggesting that the animals were still able to control the movement while performing it faster. In contrast to these effects the STN lesion induced a dramatic disturbance of the RT distribution, which was found to be scattered. Furthermore, the majority of the correct responses performed by the STN lesioned animals were found to predominantly occur on the shortest delays, thus demonstrating a tendency for the animals not to be able to wait long enough for the stimulus onset. Therefore, whereas an activation of the dopaminergic system produced essentially a motor facilitation, the STN lesion might, however, induce a more complex deficit.

In the present experiment, the histological analysis of the STN ibotenic acid lesion revealed that the medial part of the nucleus was preferentially damaged in most of the subjects. Anatomical evidence shows that the lateral part of the nucleus receives, preferentially, projections from motor cortical areas and from the "motor-related" region of the striatum, whereas the medial part of the nucleus receives projections from limbic cortical areas and limbic structures (for review, see Canteras et al., 1988; Berendse and Groenewegen, 1991; Féger et al., 1994; Wichmann et 
al., 1994a). Lesioning the medial part of the STN might therefore induce more complex ("cognitive") deficits that remain to be investigated, such as a perturbation of attentional, mnesic or time estimation processes, expressed in this task by an increase in the number of premature responses and a scattered distribution of RTs.

\section{Effects of the dopaminergic lesion after 6-OHDA infusion into the striatum}

The striatal dopamine depletion produced by the local bilateral 6-OHDA infusion in the dorsal striatum was found to induce behavioral effects opposite to those induced by the STN lesion. The impairment of the performance was mainly due to an increased number of delayed responses and a lengthening of RTs. These results are consistent with previous findings showing similar behavioral deficits in the RT task after the same dopaminergic lesion leading to $60 \%$ DA depletion in the caudal striatum (Amalric and Koob, 1987; Amalric et al., 1995). These deficits were attributed to an impairment of the initiation and probably the exccution of the movement. The striatal DA deafferentation, as assessed by the TH immunostaining, was found to be restricted to the dorsal and lateral part of the striatum. This region of the striatum receiving cortical inputs from the sensorimotor area (McGeorge and Faull, 1979) is thus supposed to be preferentially involved in the control of the conditioned movement. In the present experimental model of parkinsonism, the rats are able to perfonm the task, although with a delayed movement execution. This model appears, therefore, to be different from those leading to a massive impairment of motor control generally observed in MPTP-treated monkeys or in monoamine-depleted rodents. Furthermore, the moderate neurodegeneration of DA nerve terminals in the striatum, sparing the ascending mesocorticolimbic dopaminergic system but also the dopaminergic innervation to the STN and the GPi originating from the substantia nigra (Brown et al., 1979; Campbell et al., 1985; Parent and Smith, 1987; Lavoie et al., 1989), prevents from the occurrence of the major feeding, drinking, sensorimotor, and cognitive deficits associated with bilateral dopaminergic lesion of the forebrain bundle (Ungerstedt, 1971; Dunnett and Iversen, 1982; Salamone et al., 1990; Bergman et al., 1994). The severity of the DA depletion among these studies might thus be the critical factor in determining the beneficial effect of a subsequent STN lesion.

\section{Lesion of the STN in animals with previous dopaminergic lesion}

As expected by the behavioral effects induced by each of the lesions taken separately, the motor impairment induced by the dopaminergic lesion expressed by the number of delayed responses and the lengthening of RTs was alleviated after the lesion of the STN. Those results are in line with the previous behavioral studies showing that the lesions of the STN could reverse the motor deficits induced by MPTP treatment in monkeys (Bergman et al., 1990; Graham et al., 1990; Aziz et al., 1991; Brotchie et al., 1991; Benazzouz et al., 1993; Wichmann et al., 1994b) or dopamine depletion in rodents (Anderson et al., 1992). Alternatively, blocking the excitatory amino acid transmission at the NMDA or the AMPA receptor subtypes into the output structures of the STN was also found to restore the locomotor activity in rat and primate models of parkinsonism (Graham et al., 1990; Klockgether and Turski, 1990; Brotchie et al., 1991; Klockgether et al., 1991). Furthermore, infusion of
AMPA in the SNr was found to attenuate dopamine-dependent rotation in the rat (Burns et al., 1993). While most of these studies measured general motor activity such as locomotion or rotation in rodents or muscular rigidity and akincsia in monkeys, one study, measuring the parameters of the flexion-extension movement of the forelimb, demonstrated that the high-frequency stimulation of the STN could reverse both the muscular rigidity and the deficits in the velocity and acceleration of the movement induced by a previous unilateral MPTP treatment in monkeys (Benazzouz et al., 1993).

Whilst the long-term effect of the 6-OHDA lesion was found to recover during the last week of the experiment, the reversal of the 6-OHDA-induced deficits in the "double-lesioned" animals was expressed immediately after the STN lesion. Such a beneficial effect cannot be thus explained by the spontaneous recovery from 6-OHDA lesion by itself.

Whereas part of the present results are in general agreement with the beneficial treatment of the STN lesion in reversing the motor impairment produced by the dopaminergic lesion, the remaining deficits on the number of premature responses however, prevented the animals from returning to the baseline performance. These deficits were found to be as dramatic as in the case of the STN lesion in intact animals. This observation was confirmed by the RT distribution curve showing the same kind of scattered distribution after the STN lesion in intact- or in previously DA depleted animals. Thus, lesions of the STN in our rat model of parkinsonism do not improve the behavioral deficits as clearly as previously proposed. Since most of the previous studies referred to the effects induced by unilateral lesions of the STN (Bergman et al., 1990; Aziz et al., 1991; Anderson et al., 1992; Benazzouz, 1993; Wichmann et al., 1994b), it is thus possible that unilateral lesions could reverse the deficits induced by a dopaminergic depletion whereas the bilateral lesions of the STN could induce a deficit so severe that it could not be possibly recovered. Further studies investigating the effects of a mild STN lesion, as compared to those of the present study, on the present model of parkinsonism should help clarifying this issue. Alternatively, the 6-OHDA lesion performed into the striatum leading to a mild level of DA depletion $(<60 \%)$ in this region might be too moderate to counteract the STN lesion effects. As previously discussed, the parkinsonian model classically used (i.e., MPTP-treated monkeys or monoamine-depleted (reserpinized) rats) seemed to be more efficient to alter basal motricity, and thus might counteract all the motor abnormalities induced by the STN lesion.

Altogether, the present results show that the motor initiation deficits induced by a dopaminergic lesion of the striatum could be reversed by a bilateral ibotenic lesion of the STN, whereas deficits resulting from the STN lesion remained dramatic. Whether these deficits are purely motor or not remains to be investigated. The severity of these deficits underlines the predominant influence of the STN in controlling the behavioral motor or cognitive expression, specifically in the execution of complex conditioned tasks. Anyhow, these data question the beneficial therapeutic effects of blocking STN activity in parkinsonism.

\section{References}

Albin RL, Young AB, Penney JB (1989) The functional anatomy of basal ganglia disorders. Trends Neurosci 12:366-375.

Amalric M, Koob GF (1987) Depletion of dopamine in the caudate nucleus but not in nucleus accumbens impairs reaction-time performance in rats. J Neurosci 7:2129-2134. 
Amalric M, Moukhles H, Nieoullon A, Dazsuta A (1995) Complex deficits on reaction time performance following intrastriatal 6-OHD $\Lambda$ infusion in the rat. Eur $\mathbf{J}$ Neurosci 7:972-980.

Anderson JJ, Chase TN, Engber TM (1992) Differential effect of subthalamic nucleus ablation on dopamine D1 and D2 agonist-induced rotation in 6-hydroxydopamine-lesioned rats. Brain Res 588:307310 .

Aziz TZ, Peggs D, Sambrook MA, Crossman AR (1991) Lesion of the subthalamic nucleus for alleviation of 1-methyl-4-phenyl-1,2,3,6-tetrahydro-pyridine (MPTP)-induced Parkinsonism in the primate. Mov Disord 6:288-292.

Baunez C, Nieoullon A, Amalric M (1995) Dopamine and complex sensorimotor integration: further studies in a conditioned motor task in the rat. Neuroscience 65:375-384.

Benabid AL, Pollack P, Limousin P, Benazzouz A, Gao DM, Li J, Piallat B, Hoffmann D, LeBas JF, Iluot JC (1994) Mechanism of action of STN stimulation in alleviation of Parkinson's disease symptoms. Soc Neurosci Abstr 20:7.8.

Benazzouz A, Gross C, Féger J, Boraud T, Bioulac B (1993) Reversal of rigidity and improvement in motor performance by subthalamic high frequency stimulation in MPTP-treated monkey. Eur J Neurosci 5:382-389.

Berendse HW, Groenewegen HJ (1991) The connections of the medial part of the subthalamic nucleus in the rat: evidence for a parallel organization In: The basal ganglia III (Bernardi G, Carpenter MB, Di Chiara G, Morelli M, Stanzione P, eds). New York: Plenum.

Bergman H, Wichmann T, DeLong MR (1990) Reversal of experimental Parkinsonism by lesions of the subthalamic nucleus. Science, 249: 1436-1438.

Bergman H, Wichmann T, Karmon B, DeLong MR (1994) The primate Subthalamic nucleus. II. Neuronal activity in the MPTP model of Parkinsonism. J Neurophysiol 72:507-520.

Bernheimer H, Birkmayer W, Hornykiewicz O, Jellinger K, Seitelberger (1973) Brain dopamine and the syndromes of Parkinson and Huntington. J Neurol Sci 20:301-307.

Brotchie JM, Crossman AR (1989) Evidence for excitatory amino acid mediated subthalamic nucleus efferents in the rat. $\mathrm{Br} J$ Pharmacol 928

Brotchie JM, Mitchell IJ, Sambrook MA, Crossman AR (1991) Alleviation of Parkinsonism by antagonism of excitatory amino acid transmission in the medial segment of the globus pallidus in rat and primate. Mov Disord 6:133-138.

Brown LL, Makman MM, Wolfson LI, Dvorkin B, Warner C, Katzman $R$ (1979) A direct role of dopamine in the rat subthalamic nucleus and intrapeduncular area. Science 206:1416-1418.

Burns LH, Sato K, Wüllner U, Isacson O (1993) Intra-nigra infusion of AMPA attenuates dopamine-dependent rotation in the rat. Neuroreport 4:1075-1078.

Campbell GA, Eckardt MJ, Weight FF (1985) Dopaminergic mechanisms in the subthalamic nucleus of rat: analysis using horseradish peroxydase and microiontophoresis. Brain Res 333:261-270.

Canteras NS, Shammah-Lagnado SJ, Silva BA, Ricardo JA (1988) Somatosensory inputs to the subthalamic nucleus: a combined retrograde and anterograde horseradish peroxidase study in the rat. Brain Res 458:53-64.

Carpenter MB, Whittier JR, Mettler FA (1950) Analysis of choreoid hyperkinesia in the rhesus monkey: surgical and pharmacological analysis of hyperkinesia resulting from lesions in the subthalamic nucleus of Luys. J Comp Neurol 92:293-332.

Crossman AR (1987) Primate models of dyskinesia: the experimental approach to the study of basal ganglia related involuntary movement disorders. Neuroscience 21:1-40.

DeLong MR (1990) Primate models of movement disorders of basal ganglia origin. Trends Neurosci 13:281-285.

Dormont JF, Cheruel F, Farin D (1994) Output organization of the basal ganglia: how to explain patterns and timing of unit activity of the subthalamic nucleus and pallidum? Eur J Neurosci Suppl 7:102.

Dunnett SB, Iversen SD (1982) Sensorimotor impairments following localized kainic acid and 6-hydroxydopamine lesions of the striatum. Brain Res 248:121-127.

Féger J, Robledo P (1991) The effects of activation or inhibition of the subthalamic nucleus on the metabolic and electrophysiological activities within the pallidal complex and substantia nigra in the rat. Eur J Neurosci 3:947-952.

Féger J, Robledo P, Renwart N (1991) The subthalamic nucleus: new data, new questions. In: The basal ganglia III (Bernardi G, et al., eds). New York: Plenum.

Féger J, Mouroux M, Benazzouz A, Boraud T, Gross C, Bevan M, Crossman AR (1994) The subthalamic nucleus: a more complex structure than expected. In: The basal ganglia IV (Percheron et al., eds). New York: Plenum.

Filion M, Tremblay L (1991) Abnormal spontaneous activity of globus pallidus neurons in monkeys with MPTP-induced Parkinsonism. Brain Res 547:142-151.

Graham WC, Robertson RG, Sambrook MA, Crossman AR (1990) Injection of excitatory amino-acid antagonists into the pallidal segment of a 1-methyl-4-phenyl-1,2,3,6-tetrahydro-pyridine (MPTP) treated primate reverses motor symptoms of Parkinsonism. Life Sci 47:9197.

Hamada I, DeLong MR (1992a) Excitotoxic acid lesions of the primate subthalamic nucleus result in transient dyskinesias of the contralateral limbs. J Neurophysiol 68:1850-1858.

Hamada I, DeLong MR (1992b) Excitotoxic acid lesions of the primate subthalamic nucleus result in reduced pallidal neuronal activity during active holding. J Neurophysiol 68:1859-1866.

Kerkerian L, Salin P, Nieoullon A (1988) Pharmacological characterization of dopaminergic influence on expression of neuropeptide $\mathrm{Y}$ immunoreactivity by rat striatal neurons. Neuroscience 26:809-817.

Klockgether T, Turski L (1990) NMDA antagonists potentiate antiparkinsonian actions of L-Dopa in monoamine-depleted rats. Ann Neurol 28:539-546.

Klockgether T, Turski L, Honore T, Zhang Z, Gash DM, Kurlan R, Greenamyre JT (1991) The AMPA receptor antagonist NBQX has antiparkinsonian effects in monoamine-depleted rats and MPTP-treated monkeys. Ann Neurol 30:717-723.

Lavoie B, Smith Y, Parent A (1989) Dopaminergic innervation of the basal ganglia in the squirrel monkey as revealed by tyrosine hydroxylase immunohistochemistry. J Comp Neurol 289:36-52.

MacGeorge AJ, Faull RL (1989) The organization of the projection from the cerebral cortex to the striatum in the rat. Neuroscience 29: 503-537.

Mitchell IJ, Clarke CE, Boyce S, Robertson RG, Peggs D, Sambrook MA, Crossman AR (1989) Neural mechanisms underlying parkinsonian symptoms based upon regional uptake of 2-deoxyglucose in monkeys exposed to 1-methyl-4-phenyl-1,2,3,6-tetrahydro-pyridine (MPTP). Neuroscience 32:213-226.

Parent A, Smith Y (1987) Organization of efferent projections of the subthalamic nucleus in the squirrel monkey as revealed by retrograde labelling methods. Brain Res 436:296-310.

Paxinos G, Watson C (1986) The rat brain in stereotaxic coordinates, 2nd ed. Sydney: Academic.

Pellegrino LJ , Pellegrino AS, Cushman AJ (1979) A stereotaxic atlas of the rat brain. New York: Plenum.

Robertson RG, Farmery SM, Sambrook MA, Crossman AR (1989) Dyskinesia in the primate following injection of an excitatory amino acid antagonist into the medial segment of the globus pallidus. Brain Res 476:317-322.

Robledo P, Féger J (1990) Excitatory influence of rat subthalamic nucleus to substantia nigra pars reticulata and the pallidal complex: electrophysiological data. Brain Res 518:47-54.

Salamone JD, Zigmond MJ, Stricker EM (1990) Characterization of the impaired feeding behaviour in rats given haloperidol or dopamine-depleting brain lesions. Neuroscience 39:17-24

Sellal F, Hirsh E, Lisovoski F, Mutshler V, Collard M, Marescaux C (1992) Contralateral disappearence of parkinsonian signs after subthalamotomic hematoma. Neurology 42:255-256.

Smith Y, Parent A (1988) Neurons of the subthalamic nucleus in primates display glutamate but not GABA immunoreactivity. Brain Res, 453:353-356.

Ungerstedt U (1971) Adipsia and aphagia after 6-hydroxydopamineinduced degeneration of the nigrostriatal dopamine system. Acta Physiol Scand (Suppl) 367:96-122.

Wichmann T, Bergman H, DeLong MR (1994a) The primate subthalamic nucleus. I. Functional properties in intact animals. J Neurophysiol 72:494-506.

Wichmann T, Bergman H, DeLong MR (1994b) The primate subthalamic nucleus. III. Changes in motor behavior and neuronal activity in the internal pallidum induced by subthalamic inactivation in the MPTP model of Parkinsonism. J Neurophysiol 72:521-530.

Yamada A, Takeuchi H, Miki H (1992) Unilateral abolition of parkinsonian rigidity after subthalamic nucleus hemorrhage. Rinsho Shinkeigaku 32:887-889. 